\title{
Temporal Changes of Adsorbed Layer Thickness and Electrophoresis of Polystyrene Sulfate Latex Particles after Long Incubation of Oppositely Charged Polyelectrolytes with Different Charge Densities
}

\author{
Thi Hai Yen Doan ${ }^{1, *}$, Tien Duc Pham ${ }^{1} \mathbb{D}$, Johan Hunziker ${ }^{2}$ and Thu Ha Hoang ${ }^{3, *}$ \\ 1 Faculty of Chemistry, University of Science, Vietnam National University, Hanoi, 19 Le Thanh Tong, \\ Hoan Kiem, Hanoi 100000, Vietnam; tienducpham@hus.edu.vn or tienduchphn@gmail.com \\ 2 Graduate School of Science, Technology and Innovation, Kobe University, 1-1 Rokkodai-cho, \\ Nada-ku, Kobe 657-8501, Japan; johan.hunziker@lion.kobe-u.ac.jp \\ 3 University of Education, Vietnam National University, Hanoi, 144 Xuan Thuy, Cau Giay, \\ Hanoi 100000, Vietnam \\ * Correspondence: haiyendoan90@gmail.com or doanthihaiyen@hus.edu.vn (T.H.Y.D.); \\ hoangthuha0105@vnu.edu.vn or hoangthuha0105@yahoo.com (T.H.H.)
}

\section{check for} updates

Citation: Doan, T.H.Y.; Pham, T.D. Hunziker, J.; Hoang, T.H. Temporal Changes of Adsorbed Layer Thickness and Electrophoresis of Polystyrene Sulfate Latex Particles after Long Incubation of Oppositely Charged Polyelectrolytes with Different Charge Densities. Polymers 2021, 13, 2394. https://doi.org/ $10.3390 /$ polym 13152394

Academic Editor: Ranjit De

Received: 29 June 2021

Accepted: 18 July 2021

Published: 21 July 2021

Publisher's Note: MDPI stays neutral with regard to jurisdictional claims in published maps and institutional affiliations.

Copyright: (c) 2021 by the authors. Licensee MDPI, Basel, Switzerland. This article is an open access article distributed under the terms and conditions of the Creative Commons Attribution (CC BY) license (https:/ / creativecommons.org/licenses/by/ $4.0 /)$.

\begin{abstract}
The different desorption concepts of the two polyelectrolytes PTMA5M and PTMC5M, which have similar molecular weights and differ in the charge density on the polystyrene sulfate latex (PSL) particles by 25 times, and with various charge densities in a long incubation, were systematically investigated based on hydrodynamic adsorbed layer thickness $\left(\delta_{\mathrm{H}}\right)$ and electrophoretic mobility (EPM) under two ionic strengths in the present study. Herein, in the case of highly charged polyelectrolyte PTMA5M, desorption continued for $4 \mathrm{~h}$ and re-adsorbing proceeded after a longer incubation time higher than $4 \mathrm{~h}$. Meanwhile, in the case of lowly charged polyelectrolyte PTMC5M, an adsorption-desorption equilibrium was suggested to take into account the unchanging of both $\delta_{\mathrm{H}}$ and EPM.
\end{abstract}

Keywords: long incubation; polyelectrolytes adsorption; desorption; adsorbed layer thickness; electrophoretic mobility

\section{Introduction}

The adsorption of polyelectrolytes on solid surfaces is commonly applied to water treatment, paper manufacturing, mineral treatment, and other industrial chemical processes [1,2]. Aggregation or stability of colloidal particles can be controlled by the polyelectrolyte adsorption induced reduction or elimination of electrostatic repulsions between particles, due to the decrement or reversal of the original surface charge of particles [3-5]. In addition, the adsorption of polyelectrolytes produces an increase of adsorbed layer thickness, resulting in steric repulsion, which is responsible for the stabilization of suspensions [6]. Generally, the adsorption of polycations on an anionic surface is enhanced by the high charge density of absorbate [7], the high molecular weight of absorbate [8,9] and lower salt concentrations [10], while it is limited by some factors such as steric constraints, smaller absorbent surface charge density, or a smaller surface potential $[4,10]$.

So far, the conformation of the adsorbed polymer layer at the interface predominantly affects the flocculation or stabilization of colloidal suspensions [11-15]. The popular conformations of adsorbed polymer on the oppositely charged surface include loops, trains and tails [16-18]. The hydrodynamic adsorbed layer thickness is sensitive to the configuration of the adsorbed polymer molecules. In particular, the adsorbed layer thickness can be completely determined by the tail-conformation of the adsorbed polymer $[4,19]$. The polyelectrolytes can be initially presented as a loop- and/or tail-conformation at the 
solid-liquid interface and subsequently adopt to flatter conformations [20]. The charge density of a polyelectrolyte can modulate its conformations on oppositely charged surfaces. Hagay Kohay et al. [21] demonstrated that high-charge-density polycations prefer to directly anchor to a negatively charged montmorillonite surface in higher number of trains while low-charge-density ones preferably attach in more loops and tails. The reduction of charge density of positively charged polyelectrolyte induces that the adsorbed layer configuration on the adsorbent surface is more expanded and thicker [22,23]. The adsorbed layer thickness of low-charge-density polyelectrolytes remarkably increases with the type of surface or the higher ionic strength [10]. As a result, charged neutralization flocculation was dominant with adsorbing high-charge-density polyelectrolytes, while bridging flocculation was mainly enhanced with the adsorption of low-charge-density ones [17]. Moreover, electrophoretic mobility measurement distinguishes not only the information on the structure of the polyelectrolyte-adsorbed layer on the surface but also the roles of the electrostatic and non-electrostatic interactions in polyelectrolyte adsorption [24,25]. However, this technique has disadvantaged in order to analyze the internal structure of thick adsorbed layers [25]. Thus, an adequate complementary analytical method, such as particle tracking of Brownian motion under an optical microscope, can demonstrate adsorption kinetics of polyelectrolytes on the surface. Despite the numerous experimental and theoretical research on the effective factors underlying the adsorption and desorption polyelectrolytes with various charge density $[14,22,26]$, the temporal behaviors of those, as well as the contributions of the electrostatic and the non-electrostatic forces, have been still unclear.

In previous research, it was found that, with a short incubation of one hour, adsorption of the high-charge-density polyelectrolytes with higher molecular weights in the lower ionic strength was kinetically controlled but not in the higher ionic strength, regardless of the polyelectrolyte molecular weight $[9,26]$. The later phenomena under a high ionic strength condition have yet to be quantified. In addition, our previously published study [27] indicated that a more stable adsorbed layer of high-charge-density polyelectrolyte, which was formed closely to the core particle surface, could not be removed out of the surface, while the adsorbed layer of the low-charge-density polyelectrolyte easily removed via the perpendicular direction to the polystyrene sulfate latex (PSL) particle surface in a short incubation of one hour under the overshooting condition. Another key point that was inferred was that the stable layer immediately decided on the electrophoretic mobility (EPM). However, the mechanisms underlying different charge density polyelectrolytes on the particle surface after a long incubation time have also not been explained.

In the present study, adsorption dynamics were comprehensively solved for both highly- and lowly-charged polycations on oppositely charged PSL colloidal particles after a long incubation of 1 week. The hydrodynamic adsorbed layer thickness and electrophoretic mobility of the PSL particles with/without adsorbing polyelectrolytes were carried out as a function of time lapse under different ionic strength conditions. Based on the observed relationship between the adsorbed layer thickness and the electrophoretic mobility, different behaviors of polyelectrolytes differing of the charge density on the PSL colloidal particles under long incubation are also proposed.

\section{Materials and Methods}

\subsection{Materials}

In this study, polystyrene sulfate latex particles (PSL, Thermo Fisher Scientific, Inc., Waltham, MA, USA) with a diameter of about $1.2 \pm 0.01 \mu \mathrm{m}$ and a surface charge density of $5.5 \mu \mathrm{C} \cdot \mathrm{cm}^{-2}$ were employed as negatively charged colloidal particles. Before each adsorption experiment, sonication of the PSL colloidal particles was conducted for $25 \mathrm{~min}$ to eliminate particle aggregations. The PSL colloidal particles with initial concentration of $10^{8}$ particles $\cdot \mathrm{cm}^{-3}$ and initial concentration of particle sulfate groups of $1.8 \times 10^{14} \mathrm{~cm}^{-3}$ were applied in all experiments. Two linear polycations with similar molecular weight and a charge density difference of 25 times were used as absorbates in adsorption mea- 
surements. The polyelectrolytes are poly((2dimethylamino) ethyl methacrylate) methyl chloride quaternary salt (PTMA5M; Kaya Floc Co. Ltd., Tokyo, Japan) and a co-polymer of PTMA5M and acrylamide (PTMC5M; Kaya Floc Co. Ltd.) corresponding to similar nominal molecular weights of $4.9 \times 10^{6}$ and $5.2 \times 10^{6} \mathrm{~g} \cdot \mathrm{mol}^{-1}$. The charge densities, $\rho$ of PTMA5M and PTMC5M were $100 \%$ and $4 \%$, respectively. The chemical structures of two polycations were described in [27]. Stock polyelectrolyte solutions of $100 \mathrm{mg} \cdot \mathrm{L}^{-1}$ were prepared by stirring an appropriate amount of polycations with potassium chloride solutions for $72 \mathrm{~h}$. Working solutions diluted from the stock solutions were used for a week. All polyelectrolyte solutions were kept at approximately $5{ }^{\circ} \mathrm{C}$ in darkness to eliminate the light exposure-induced degradation. Potassium chlorine solutions at the concentration of 0.1 and $10 \mathrm{mM}$ were applied to maintain the ionic strength. Before conducting the experiments, the electrolyte solutions were filtered by using a $0.2 \mu \mathrm{m}$ cellulose membrane. Ultrapure water produced from Elix Advantage 5 (Millipore) with electric conductivity around $0.6 \mu \mathrm{S} \cdot \mathrm{cm}^{-1}$ was employed in all solutions and measurements.

\subsection{Methods}

Determination of hydrodynamic layer thickness was by mean of particle tracking of Brownian motion.

The Brownian movements of single PSL colloidal particles with/without adsorption of polyelectrolytes in one dimension were tracked by using a CCD video camera (WVBL200, Matsushita Co. Ltd., Yamanashi, Japan) equipped with an optical microscope (BX50, Olympus). The method was described in more detail in [26,27]. The diffusion coefficient of single PSL particle $D$ was determined using the Einstein relation [26,28]:

$$
\overline{\Delta x^{2}}=2 D \tau
$$

where $\overline{\Delta x}(\mathrm{~m})$ is the particle displacement calculated from the center of mass at time interval of $\tau$ (s).

Then hydrodynamic layer thickness of adsorbed polyelectrolytes was calculated by comparing $D$ of the bare single PSL particle with that of a particle with adsorbing polyelectrolyte using the Stokes-Einstein equation, as shown in the following Equations (2) and (3) below, respectively.

$$
\begin{gathered}
D=\frac{k_{B} T}{6 \pi \alpha \mu} \\
D=\frac{k_{B} T}{6 \pi(\alpha+\delta H) \mu}
\end{gathered}
$$

where $k_{B}$ is Boltzmann constant $\left(k_{B}=1.38 \times 10^{-23} \mathrm{~J} \cdot \mathrm{K}^{-1}\right)$, T is the absolute temperature $(\mathrm{K}), \alpha$ is hydrodynamic radius $(\mathrm{m}), \mu$ is the solvent viscosity (Pa.s) and $\delta_{H}(\mathrm{~m})$ is the hydrodynamic layer thickness of adsorbed polyelectrolytes.

\subsection{Experimental Procedure}

A polycation volume of $5.0 \mathrm{~mL}$ under two ionic strength conditions was added to one side of a forked flask while in the other side, the same volume of the initial PSL concentration of $10^{8}$ particles $\mathrm{cm}^{-3}$ was introduced. An end-over-end apparatus described in the previous reference [9] was used to mix the samples for 10 times at a frequency of $1 \mathrm{~Hz}$ (Figure 1). The experiment procedure was mentioned in more detail in [27]. Lapse of time was calculated from stopping mixing the samples. The experimental measurements were carried out at room temperature $(293 \pm 2 \mathrm{~K})$, controlled with an air conditioner.

2.3.1. Experimental Measurements of Hydrodynamic Layer Thickness of Adsorbed Polyelectrolytes

After mixing and fixed incubation time, the samples were added to a $0.2 \mathrm{~mm}$ width $\times 2.0 \mathrm{~mm}$ length rectangle capillary tube. The Brownian movements of a single colloidal PSL particle were surveyed by using an optical microscope with a magnitude of $80 \times$ and 
a time resolution of $0.1 \mathrm{~s}$. The video of Brownian movements of a colloidal particle was recorded with 500 frames and 1000 displacements for about $0.033 \mathrm{~s}$. The clear images of the PSL particle were maintained by the manual microscope focus.

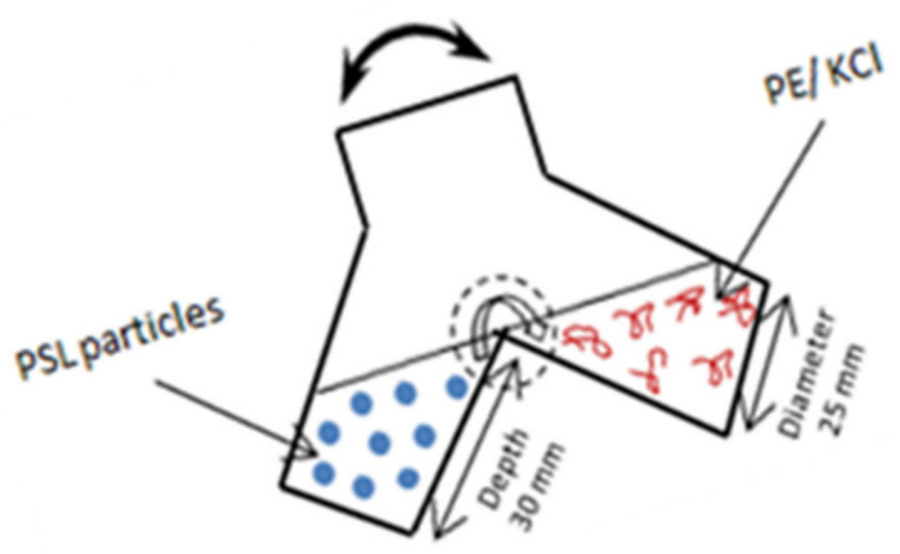

Figure 1. Schematic illustration of an end-over-end apparatus used for mixing samples.

\subsubsection{Electrophoretic Mobility Measurements}

The electrophoretic mobility of PSL particles $(\mathrm{EPM})\left(\mu \mathrm{m} \cdot \mathrm{cm} \cdot \mathrm{V}^{-1} \cdot \mathrm{s}^{-1}\right)$ was measured in an electric field of $11.3\left(\mathrm{~V} \cdot \mathrm{cm}^{-1}\right)$ by the Zeta-sizer Nano-ZS setup, with a laser velocimetry (Malvern Instruments Ltd., Worcestershire, UK). The samples were introduced into a plastic capillary cell and then inserted in the analyzer. Each sample was measured for at least 3 times. Each measurement was carried out with 11 sub-runs.

\subsubsection{Viscosity Measurements}

The viscosity of the solutions was determined by a Cannon-Fenske capillary viscometer (S0-94331, Model 100, SIBATA Ltd., Saitama, Japan). The viscosity experiments were conducted at $293 \mathrm{~K}$ using a controlled temperature bath. The sample was added into a viscometer, inserted to the bath, and stood for $5 \mathrm{~min}$ to get a temperature equilibration before starting each experimental measurement. Relative viscosity, $\eta_{r}$ was determined by comparing the time of polymer solution, $t_{s}$, and the time of solvent, $t_{0}$, flowing through the viscometer.

$$
\eta_{r}=\frac{t_{s}}{t_{0}}
$$

At low polyelectrolyte concentration, polyelectrolyte existed as a random coil in the solution and assumed as a considerable sphere. The charge density of electrolyte ions was $1000 \mathrm{mmol} \cdot \mathrm{L}^{-1}$ at the $\mathrm{KCl}$ concentration of $1 \mathrm{M}$, while the charge densities of PTMA5M and PTMC5M calculated were approximately 569.02 and $13.41 \mathrm{mmol} \cdot \mathrm{L}^{-1}$, respectively, at $5 \mathrm{ppm}$ of polyelectrolyte concentration. Therefore, the polyelectrolyte charges were compensated by oppositely charged electrolyte ions, considering as a neutral particle in the solution. The size of the polyelectrolyte was calculated by Einstein's viscosity equation, applied for neutral spherical particles [29]. The Einstein's equation is given as the following (5):

$$
\eta_{r}=\frac{\eta_{s}}{\eta_{0}}=1+\frac{5}{2} \Phi
$$

where $\eta_{s}$ is the viscosity of polymer solution $\left(\mathrm{N} \cdot \mathrm{s} \cdot \mathrm{m}^{-2}\right), \eta_{0}$ is the solvent viscosity $\left(\mathrm{N} \cdot \mathrm{s} \cdot \mathrm{m}^{-2}\right)$, $\Phi$ is the volume fraction.

Then, the hydrodynamic diameter of polymer was calculated by:

$$
\left.d=10^{9} \times\left[\frac{\left(\eta_{r}-1\right) \times M_{w}}{\frac{5}{2} \times\left(\frac{\pi}{6}\right) \times C \times N_{A}}\right)\right]^{\frac{1}{3}}
$$


where $d$ is the hydrodynamic diameter of polymer in solution (nm), $M_{w}$ is the molecular weight of polymer $\left(\mathrm{g} \cdot \mathrm{mol}^{-1}\right), C$ is the polymer concentration (ppm), $N_{A}$ is the Avogadro number $\left(N_{A}=6.02 \times 10^{23} \mathrm{~mol}^{-1}\right)$.

\section{Results and Discussion}

3.1. Contributions of Electrostatic and Non-Electrostatic Interactions on Adsorption of Polyelectrolytes with Different Charge Densities onto PSL Particles at Different Ionic Strengths

Adsorption of polyelectrolytes with high- and low-charge densities onto the positively charged PSL particles immediately occurred when polycations were added to the colloidal suspension. The primary driving forces for the adsorptions of PTMA5M and PTMC5M are mainly electrostatic attractions between particle surface and oppositely charged polyelectrolyte segments $[17,30,31]$ and non-electrostatic interactions such as hydrogen bonding, hydrophobic interaction, and ion bindings [24,32,33]. Furthermore, the non-electrostatic interactions between polyelectrolyte segments can be attributed to an increase layer thickness, which continues after reaching an isoelectric point (IEP) [24]. It can be inferred that the hydrodynamic diameter of the particle increases with the decreasing absolute electrophoretic mobility of particles, and this even leads to a reversal of particle charge when adsorbing an excess dosage of polycations (Figure 2). This phenomenon is also clearly affirmed in the previous research [27]. As a result, the enhancement of aggregation, or the stability of the colloidal suspension, occurs after adding the positively charged polyelectrolytes to the oppositely charged PSL particles. The increments in hydrodynamic particle diameter due to adsorption of polyelectrolytes were evaluated by calculating the difference between the diffusion constant of a bare PSL particle and another single PSL particle with the adsorbed polycations.

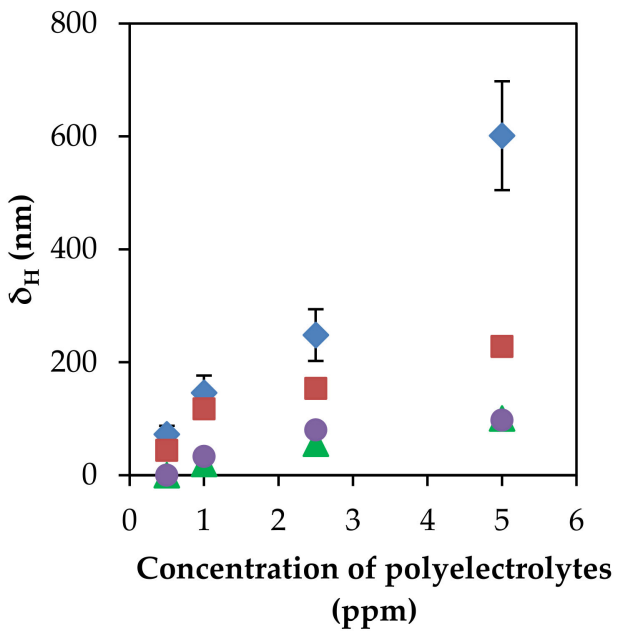

(a)

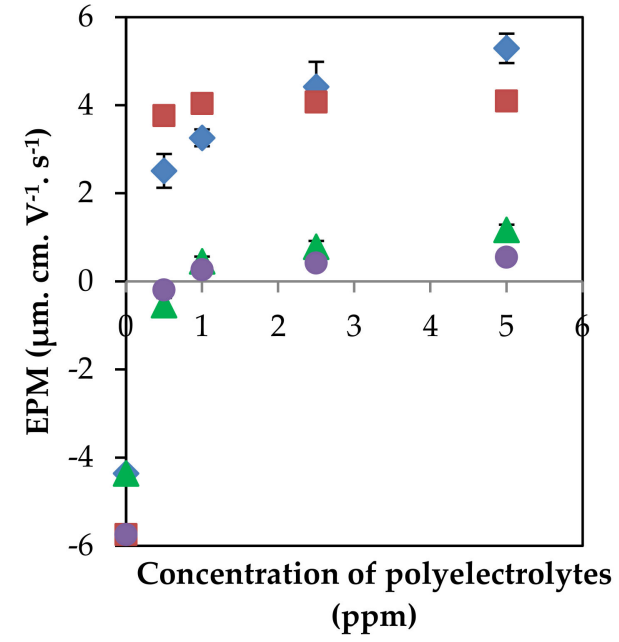

(b)

Figure 2. Variation of: (a) the hydrodynamic-adsorbed layer thickness, $\delta_{\mathrm{H}}$ and (b) the electrophoretic mobility of polyelectrolyte-adsorbed PSL particles, EPM acting as a function of the polyelectrolyte dosage after $5 \mathrm{~min}$ of incubation at different salt concentrations, including $0.1 \mathrm{mM} \mathrm{KCl}$ : PTMA5M


deviations of at least three different runs.

Figure 2 shows that, at a fixed ionic strength, the adsorbed layer thickness as well as the EPM after 5 min of incubation dramatically increased when increasing the initial polyelectrolyte concentration from 1 to $10 \mathrm{ppm}$. This increment could be due to kineticallycontrolled adsorption and/or either a dynamic adsorption-desorption equilibrium or a partial surface coverage $[19,34,35]$. At the beginning, a higher concentration of polyelec- 
trolytes in the solution was more numerous as polyelectrolytes diffused from the solution to the PSL particle surface $[19,35]$. This led to polyelectrolyte attachments to the particle surface, consisting of adsorbed polyelectrolyte chains with new conformations [32], and further resulting in an increment of adsorbed layer thickness. On the other hand, the value of IEP extrapolated from Figure 2 was approximately 0.28 and $0.29 \mathrm{ppm}$ in the case of the high-charge-density polyelectrolyte PTMA5M, while the value was about 0.6 and $0.8 \mathrm{ppm}$ in the case of low-charge-density polyelectrolyte PTMC5M, at high and low ionic strengths, respectively. In the range of the initial polyelectrolyte concentration of 2 to $10 \mathrm{ppm}$, the total adsorbed polyelectrolyte charges over-compensated the charges of the particle surface. Thus, the explanation of a polyelectrolyte partially-coated particle surface was excluded. Moreover, the IEP value of the highly charged polyelectrolyte was lower than the lowly charged one, due to a faster adsorption process of PTMA5M, resulting in more than a 25-fold higher charge density than PTMC5M. These results are in agreement with the almost previous findings [22,23].

Considering the polyelectrolytes adsorption on an oppositely charged surface driven by the electrostatic interactions, the adsorption regimes [36] were limited by the shielding that induced by not only electrostatic repulsions between the positively charged groups in polyelectrolyte chains but also in the electrostatic polyelectrolyte segment-PSL surface attractions when the ionic strength increased. In the former effect, polyelectrolytes shrunk in correlation with a reduction of relative viscosity when the $\mathrm{KCl}$ concentration increased from $0.1 \mathrm{mM}$ to $1 \mathrm{M}$ (Table 1). It should be noted that polyelectrolytes are less coiled in solution, inducing less swollen adsorbed layer. However, this effect was negligible for the low-charge-density polyelectrolyte because of a significant distance between the charged groups on the polyelectrolyte chain (approximately $25 \mathrm{~nm}$ ). Therefore, the relative viscosity of PTMC5M measured as well as the hydrodynamic thickness of adsorbed layer had not much difference between all ionic strengths. In the lateral effect, the attractive interactions, not only between the polyelectrolyte segments and the PSL particles, but also between polyelectrolyte chains, were all reduced, corresponding to a reduction of $\delta_{H}$ as well as the PSL surface charge, as evidenced by a decrease in the EPM. The tendency of the attractive forces to ionic strength was similar to polyelectrolyte adsorption on the surface with opposite charge [37]. These results suggest that the electrostatic attraction is the exclusive driving force for the adsorption of the highly positively charged PTMA5M onto the oppositely charged PSL surface.

Table 1. Relative viscosity and hydrodynamic diameter of PTMA5M and PTMC5M at 5 ppm determined by Einstein's equation at different $\mathrm{KCl}$ concentrations [27]. The standard deviations were calculated by at least eight experiments.

\begin{tabular}{|c|c|c|c|c|}
\hline \multirow{2}{*}{ Polyelectrolytes } & \multicolumn{3}{|c|}{ Relative Viscosity } & \multirow{2}{*}{$\begin{array}{c}\begin{array}{c}\text { Hydrodynamic } \\
\text { Diameter (nm) }\end{array} \\
1 \mathrm{M} \mathrm{KCl}\end{array}$} \\
\hline & $0.1 \mathrm{mM} \mathrm{KCl}$ & $10 \mathrm{mM} \mathrm{KCl}$ & $1 \mathrm{M} \mathrm{KCl}$ & \\
\hline PTMA5M, $\rho=100 \%$ & $1.0260 \pm 0.0012$ & $1.0089 \pm 0.0009$ & $1.0030 \pm 0.0005$ & $155.46 \pm 8.97$ \\
\hline PTMC5M, $\rho=4 \%$ & $1.0063 \pm 0.0008$ & $1.0062 \pm 0.0007$ & $1.0038 \pm 0.0004$ & $171.91 \pm 6.69$ \\
\hline
\end{tabular}

However, contrary to the tendency of the highly charged polyelectrolyte PTMA5M, it can be observed in Figure 2 that there was an increase in the adsorbed layer thickness of the lowly charged polyelectrolyte PTMC5M, increasing the ionic strength. PTMC5M even adsorbed continuously after reaching the IEP. It was concluded that there are additional nonelectrostatic interactions, not only between the PSL particle surface and the polyelectrolyte segments, but also between polyelectrolyte chains $[37,38]$. The non-electrostatic forces were very important for the adsorption process of low-charge-density polyelectrolytes [33]. Some authors also found that the thicker adsorbed layer was plotted as a function of increment of the Debye-Hückel parameter, $\mathrm{K}^{-1}[36,39,40]$. It could be suggested that not only were the hydrophobic interactions between surface and polyelectrolytes promoted, 
but the repulsive forces between polyelectrolyte segments were also reduced when the ionic strength increased. This resulted in the main increment of the adsorbed layer thickness, as well as the more and/or longer extended polyelectrolyte conformation of protruding loops and tails into the solution $[4,38]$. Therefore, the non-electrostatic force plays a significantly important role in the PTMC5M adsorption, resulting simultaneously in attaching loosely to the PSL particle surface and strongly to other PTMC5M, as well as exhibiting a swelling conformation when the ionic strength increases. Therefore, the adsorbed amount of the lowcharge-density polyelectrolyte was reduced upon addition of salt in almost of cases [10].

The sign of PSL electrophoretic mobility changed from negative to positive, passing through the IEP. These results from the adsorption of the oppositely charged polyelectrolytes are illustrated in Figure 2. A shift in the IEP can be observed as the concentration of $\mathrm{KCl}$ increased 100 times from 0.1 to $10 \mathrm{mM}$. The IEP value shifted to a higher value as the ionic strength increased, proving the limited adsorption of PTMA5M. Oppositely, the lower IEP value at the higher ionic strength confirms that more PTMC5M adsorbed onto the PSL colloidal suspension due to promotion of hydrophobic interactions under the high ionic strength condition.

\subsection{Relaxation Behaviors of Adsorbed Layer of Polyelectrolytes with Different Charge Density on the PSL Particles in a Long Incubation}

In our previous research [27], the non-equilibrium states of various charge density polycations on the negatively charged PSL colloidal particles were clarified. It was confirmed that the $\delta_{\mathrm{H}}$ of PTMA5M on the PSL particle surfaces decreased while EPM of PTMA5M-adsorbed PSL particles kept constant under the overshooting condition in a short incubation of $1 \mathrm{~h}$. Meanwhile, the $\delta_{\mathrm{H}}$ of PTMC5M maintained while the EPM decreased slightly with an excess of PTMC5M dosage in the time scale of $1 \mathrm{~h}$. Moreover, the different concepts corresponding with the desorption of the different-charge-density polyelectrolytes were proposed. That is, the high-charge-density polyelectrolyte PTMA5M chains were desorbed sequentially from the outer layer to the inner layer (which is closer to the PSL particle core), while the low-charge-density polyelectrolyte PTMC5M chains perpendicularly aligned to the PSL surface were removed. In the present study, the behaviors of the positively charged polyelectrolytes with various charge densities on the PSL particle surface in a long incubation from $1 \mathrm{~h}$ to 1 week were thoroughly investigated.

At the given polyelectrolyte concentration, the $\delta_{\mathrm{H}}$ and the EPM values were changed against the time lapse in the time scale of 1 week at both ionic strengths. There are herein some different tendencies. In the case of PTMA5M adsorption, the $\delta_{\mathrm{H}}$ dramatically decreased from 1 to $4 \mathrm{~h}$ of the time lapse in two ionic strengths, while the value of EPM changed insignificantly (Figure 3). Re-conformation and/or detachment of polyelectrolytes might explain the decrement of the adsorbed layer thickness [20,41]. The EPM clearly suggests that the concepts could be responsible for this decrement of the adsorbed layer thickness. According to Ohshima and Kondo's theory, the electrophoretic mobility could depend on total charge amount contained inside the whole adsorbed layer, the ionic shielding effect of electrolytes, the adsorbed layer thickness $\left(\delta_{\mathrm{H}}\right)$, and a uniform density of fixed-charge groups $\left(\rho_{\text {fix }}\right)$ distributed inside the adsorbed layer [42-47]. If the adsorbed polyelectrolytes were re-conformed on the PSL particle surface, the total amount of charges remained constant, while the area in which fewer electrolyte ions penetrated was restricted, as the adsorbed thickness changed into a flatter-conformation, decreasing the electrolyte ion shielding effect [46]. As a result, the electrophoretic mobility could increase.

Herein, the EPM did not change significantly in the range of time lapse from 1 to $4 \mathrm{~h}$, suggesting a desorption process. Moreover, there was a significant increment of the $\delta_{\mathrm{H}}$ after $4 \mathrm{~h}$ of incubation, potentially due to re-adsorption, as the remaining PTMA5M molecules were still not eliminated from the solution. Meanwhile, the EPM remained constant at two ionic strengths. This tendency of the EPM after $4 \mathrm{~h}$ incubation could be explained by the fixed charge group density distributed inside adsorbed layer and/or the electrolyte ion shielding effect. As immediately mentioned above, the more penetrative the electrolyte ions were during the increment of the $\delta_{\mathrm{H}}$, the stronger the shielding effect of electrolyte 
ions was. As a result, the value of the EPM ought to decrease. However, the EPM remained unchanged against the significant changes of the $\delta_{\mathrm{H}}$. It was inferred that the shielding effect of the electrolyte ions was negligible, hence the EPM was totally dependent on the $\rho_{\text {fix }}$ and remained. Summarily, desorption and re-adsorption takes account for the decrement and the increment of the $\delta_{\mathrm{H}}$ in a short incubation lower than $4 \mathrm{~h}$ and a long incubation higher than $4 \mathrm{~h}$, respectively, while the fixed charge groups inside the PTMA5M-adsorbed layer resulted in a constant EPM value.

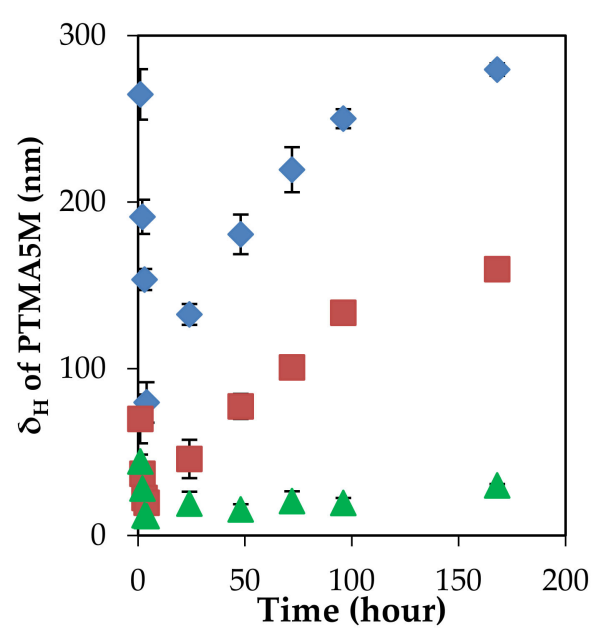

(a)

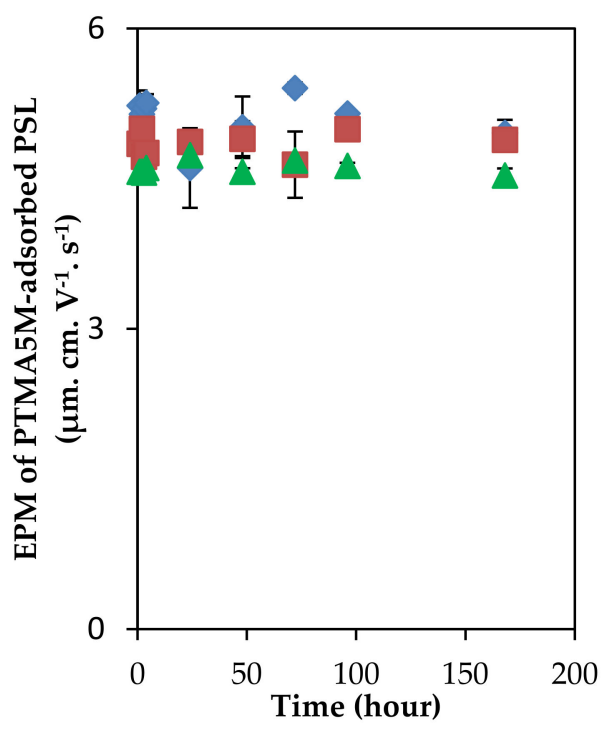

(c)

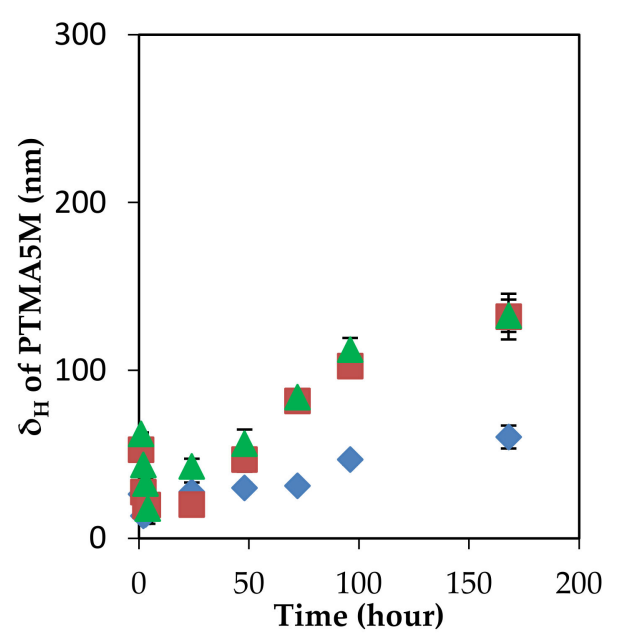

(b)

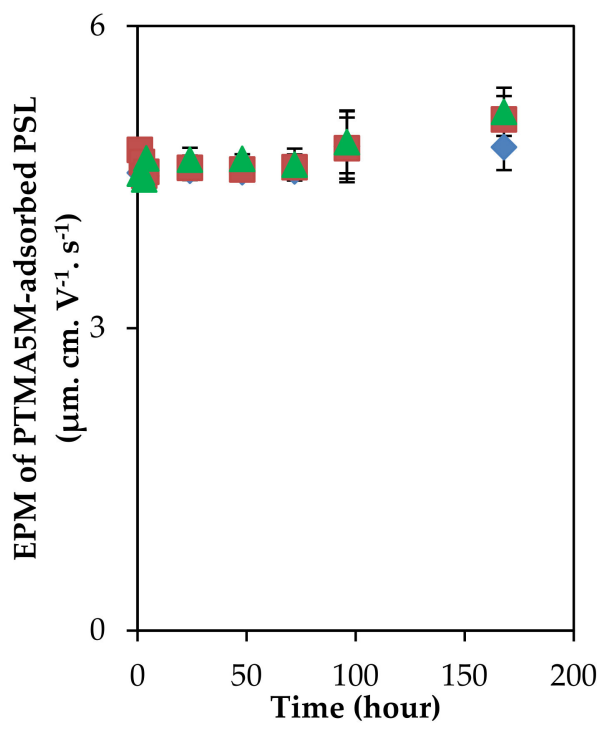

(d)

Figure 3. $\delta_{\mathrm{H}}$ and EPM of PTMA5M-adsorbed PSL colloidal particles as a function of time lapse under two ionic strength conditions. In $0.1 \mathrm{mM} \mathrm{KCl}$ : (a) $\delta_{\mathrm{H}}$, (c) $\mathrm{EPM}$ and in $10 \mathrm{mM} \mathrm{KCl}$ : (b) $\delta_{\mathrm{H}}$, (d) EPM. Initial PTMA5M concentrations: 10 ppm ( $), 5$ ppm ( $\square$ ), 2 ppm ( $\Delta$ ). The deviations of different runs are shown by the error bars.

In addition, the mobility trend could be explained on the basis of the dependence of the electrophoretic mobility of soft particles on the ratio of adsorbed layer thickness and the Debye length $\left(\delta_{\mathrm{H} \cdot \mathrm{K}}\right)[42,45]$. When the ratio $\left(\delta_{\mathrm{H} \cdot \mathrm{K}}\right)$ was higher than unity at both ionic strengths $\left(\delta_{\mathrm{H}} \cdot \mathrm{K}>>1\right)$, the EPM mainly depended on the uniform density of fixed-charge groups within the polyelectrolyte layer, and was insensitive to the adsorbed layer thickness. In the case of PTMC5M adsorption, both the adsorbed layer and the EPM remained constant 
in a long incubation of 1 week under two ionic strength conditions (Figure 4). One more time, desorption could affirm to be a reason. The low-charge-density polyelectrolytes easily detached perpendicularly from the PSL surface, introducing the constant adsorbed layer thickness and the decrement of the EPM in the short incubation of $1 \mathrm{~h}$, observed in our previous study [27]. However, herein, the EPM remained constant in the longer incubation. After the long incubation, it can be suggested that an adsorption-desorption equilibrium was reached. As a result, a fixed value of the $\delta_{\mathrm{H}}($ about $100 \mathrm{~nm})$ was obtained while the fixed-charge groups inside the adsorbed layer were responsible for the uncharged value of the EPM.

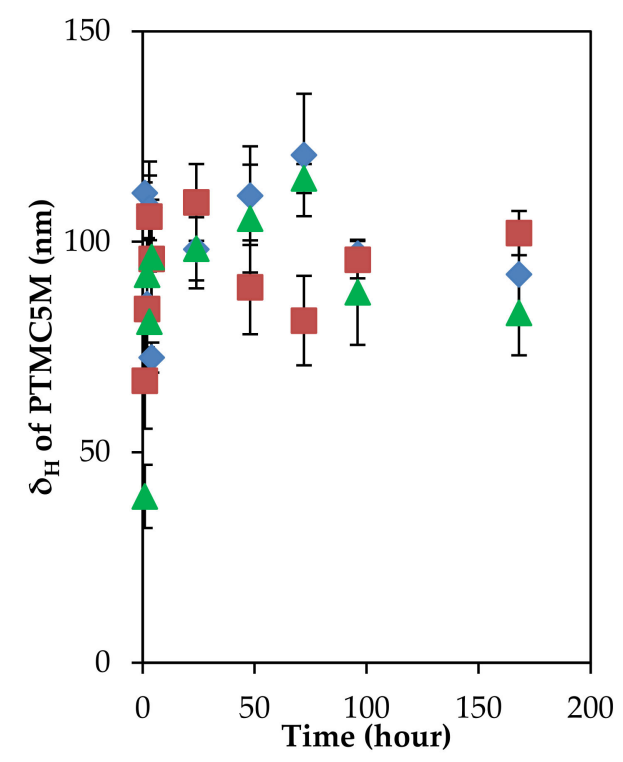

(a)

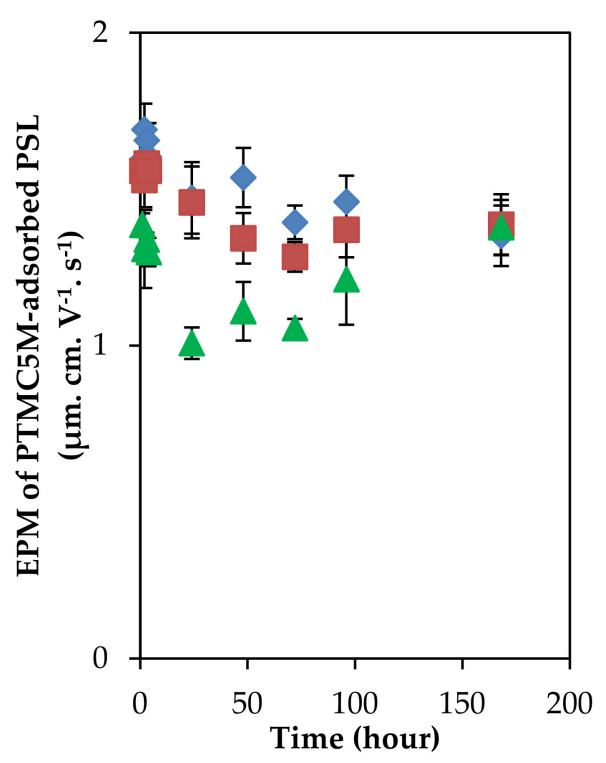

(c)

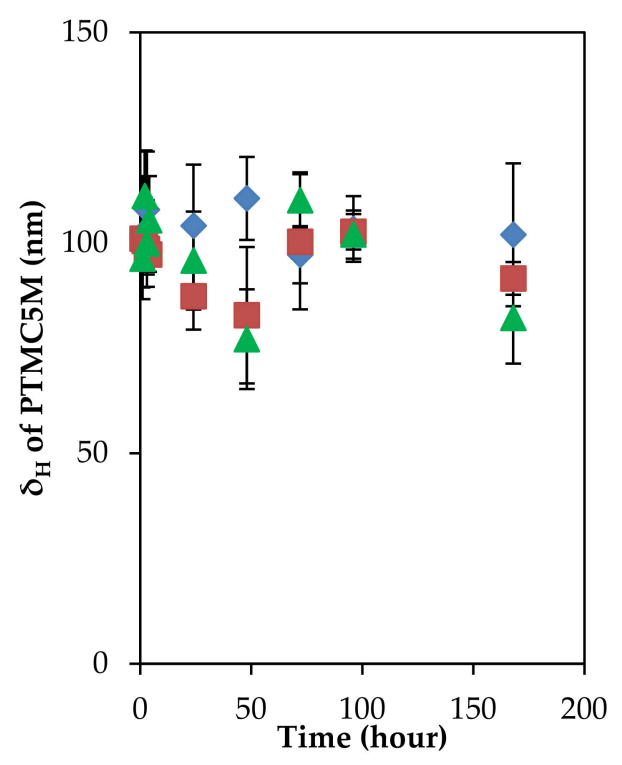

(b)

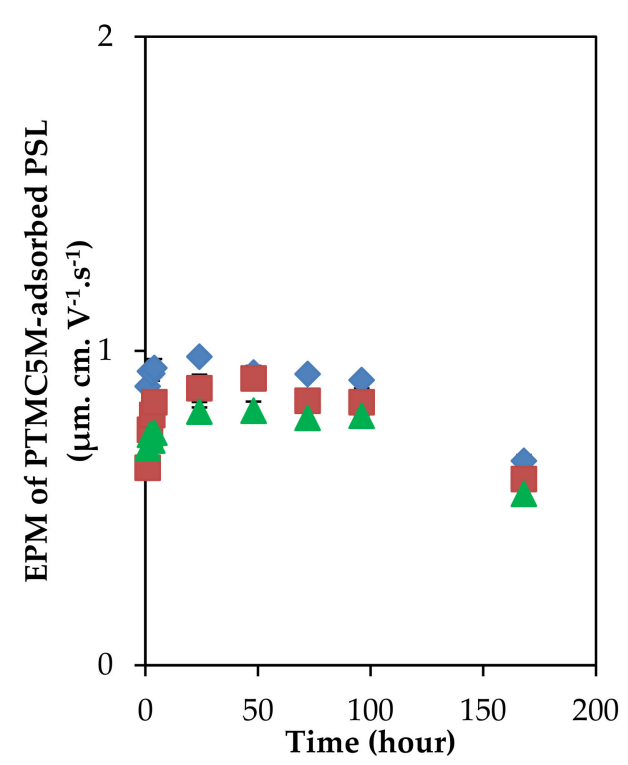

(d)

Figure 4. $\delta_{\mathrm{H}}$ and EPM of PTMC5M-adsorbed PSL colloidal particles as a function of time lapse under two ionic strength conditions. In $0.1 \mathrm{mM} \mathrm{KCl:} \mathrm{(a)} \delta_{\mathrm{H}},(\mathbf{c}) \mathrm{EPM}$ and in $10 \mathrm{mM} \mathrm{KCl}$ : (b) $\delta_{\mathrm{H}}$, (d) EPM. Initial PTMC5M concentrations: 10 ppm ( $), 5$ ppm ( $\square), 2$ ppm ( $\mathbf{\Delta})$. The deviations of different runs are shown by the error bars show. 
3.3. Adsorption/Desorption Concepts of Polyelectrolytes with Different Charge Density on the PSL Particles in a Long Incubation

Two previously proposed concepts for the desorption of two polyelectrolytes with different charge densities on the PSL colloidal particles in the short incubation of $1 \mathrm{~h} \mathrm{[27]}$ were affirmed by the data found in the present study. However, the adsorption behaviors of the different charge density polycations on the oppositely charged PSL particles with a long incubation of 1 week were systematically investigated, based on the changes of the hydrodynamic adsorbed layer thickness and the EPM. Formerly, the detachment of the homogenous adsorbed layer of PTMA5M from the outer to the inner took into account the simple reduction of thickness, while the fixed-charge groups responded to the remaining EPM of PTMA5M adsorbed-PSL particles through $4 \mathrm{~h}$ under two ionic strength conditions. When the adsorption was mainly driven by the electrostatic interactions, i.e., PTMA5M, the inner-adsorbed layer, which is close to the PSL surface, tightly attached, while the outer-adsorbed layer of loose attachments to the particle surface easily desorbed. This results from the weaker non-electrostatic interaction between polyelectrolyte segments, rather than the electrostatic ones between particles and polyelectrolytes. The desorption process continued happening until reaching a fixed $\delta_{\mathrm{H}}($ about $20 \mathrm{~nm}$ ), suggesting the presence of a thin, stiff adsorbed layer. A long desorption process of PTMA5M (about $4 \mathrm{~h}$ ) was suggested. Moreover, the re-adsorption occurred after $4 \mathrm{~h}$ of incubation, as the free PTMA5M was still not removed from the solution, inducing the increment of the $\delta_{\mathrm{H}}$. The mobility was constant in the long incubation of 1 week due to the $\rho_{\text {fix }}$. The schematic behaviors of the high-charge-density PTMA5M on the PSL colloidal particles with the negative charges are illustrated in Figure 5.
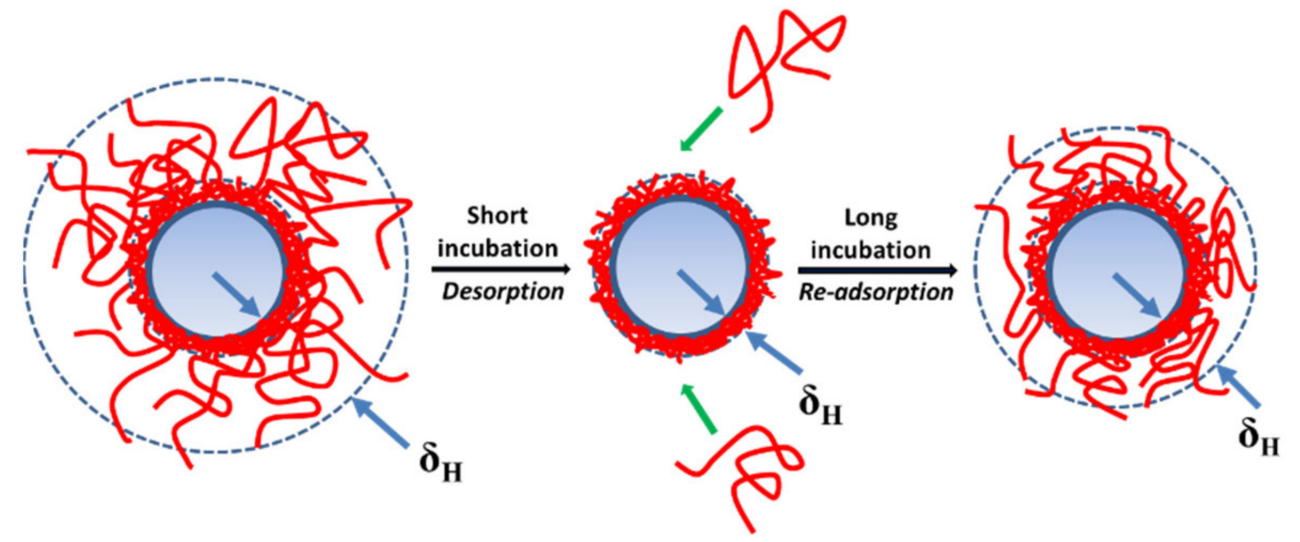

Figure 5. The polycation PTMA5M behaviors on the PSL colloidal particles with opposite charge in the long incubation.

Latterly, in the PTMC5M adsorption of the short incubation of $1 \mathrm{~h}$, the removal of alternative parts of the adsorbed polyelectrolyte from the PSL surface introduced the decrement of the average charge density and a constant thickness. Herein, some parts of PTMC5M weakly made contact with the PSL surface due to hydrophobic interactionsdesorption occurred easily. In the long incubation of more than $1 \mathrm{~h}$, it was suggested that an adsorption-desorption equilibrium was reached in the case of PTMC5M adsorption. Then, the fixed values of both the adsorbed layer thickness and the EPM were reached. The schematic behaviors of polycation PTMC5M on the PSL surface are illustrated in Figure 6. 

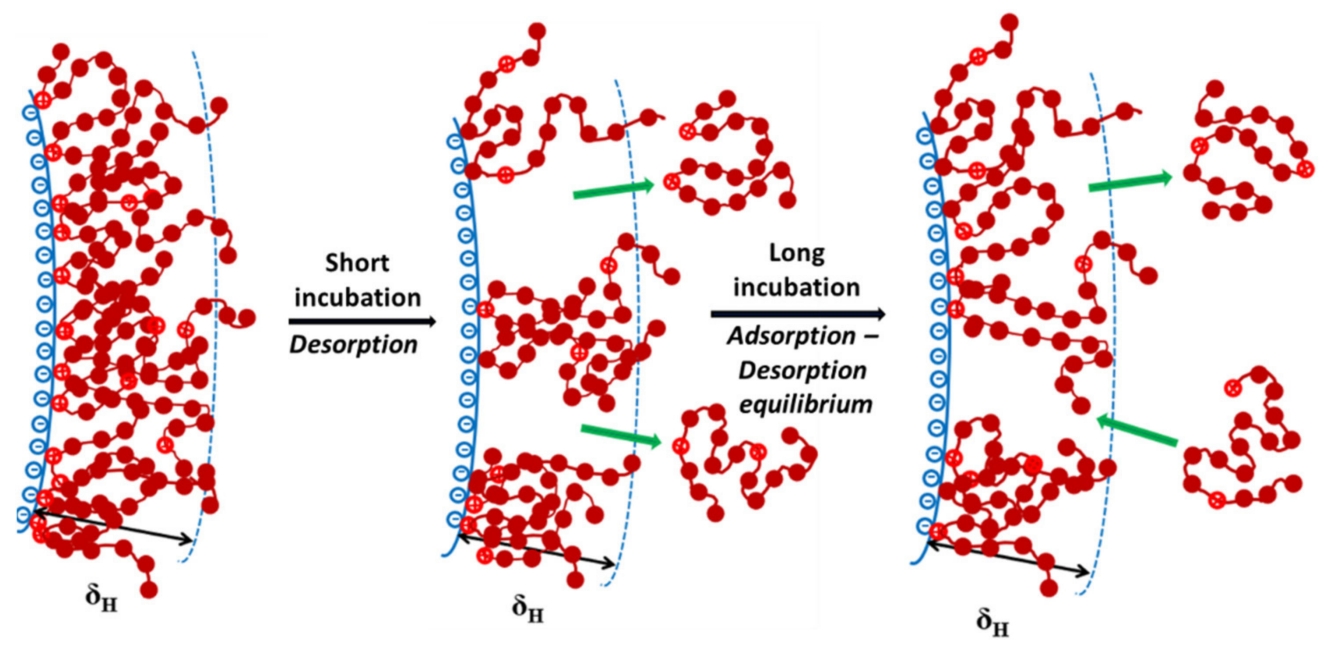

Figure 6. The polycation PTMC5M behaviors on the PSL colloidal particles with opposite charge in the long incubation.

\section{Conclusions}

The adsorption behaviors of polycations, with 25-fold differences in charge density and with similar molecular weights, onto oppositely charged PSL particle surfaces were comprehensively investigated in this study. This included the temporal changes of the hydrodynamic-adsorbed layer thickness and the electrophoretic mobility under the different ionic strength conditions, with a long incubation of 1 week. In the case of the adsorption of the highly charged polyelectrolyte PTMA5M, fast adsorption occurred at initial times, followed by desorption for about $4 \mathrm{~h}$, and finally a re-adsorption process was observed. On the other hand, an adsorption-desorption equilibrium was suggested in the case of the lowly charged polyelectrolyte PTMAC5M. It resulted in a fixed value of the adsorbed layer and a constant mobility. The value of electrophoretic mobility followed Ohshima's theory and was dependent on the fixed charge groups within the adsorbed layer and/or the electrolyte ion effect.

Author Contributions: Conceptualization, T.H.Y.D. and T.H.H.; methodology, T.D.P. and T.H.Y.D.; software, J.H. and T.H.Y.D.; validation, T.D.P. and T.H.H.; formal analysis, T.H.Y.D. and T.D.P.; investigation, J.H. and T.H.H. and T.H.Y.D.; resources, J.H. and T.D.P.; data curation, T.H.Y.D. and T.D.P.; writing —original draft preparation, T.H.Y.D., T.H.H. and J.H.; writing-review and editing, T.H.Y.D., J.H. and T.D.P.; visualization, T.H.H. and T.D.P.; supervision, T.D.P. and T.H.H.; project administration, T.H.H.; funding acquisition, T.H.Y.D. All authors have read and agreed to the published version of the manuscript.

Funding: This research received no external funding.

Institutional Review Board Statement: Not applicable.

Informed Consent Statement: Not applicable.

Data Availability Statement: Not applicable.

Acknowledgments: The authors would like to thank Yasuhisa Adachi, University of Tsukuba, Japan for the chemical and equipment supports and Christopher J. Vavricka, Kobe University, Japan for critical reading to improve English.

Conflicts of Interest: The authors declare no conflict of interest. 


\section{References}

1. Moody, G.M. Polymeric Flocculants. In Handbook of Industrial Water Soluble Polymers; John Wiley \& Sons: Hoboken, NJ, USA, 2007; pp. 134-173.

2. Nasser, M.S.; Twaiq, F.A.; Onaizi, S.A. Effect of polyelectrolytes on the degree of flocculation of papermaking suspensions. Sep. Purif. Technol. 2013, 103, 43-52. [CrossRef]

3. Dickinson, E.; Eriksson, L. Particle flocculation by adsorbing polymers. Adv. Colloid Interface Sci. 1991, 34, 1-29. [CrossRef]

4. Rojas, O.J.; Claesson, P.M.; Muller, D.; Neuman, R.D. The Effect of Salt Concentration on Adsorption of Low-Charge-Density Polyelectrolytes and Interactions between Polyelectrolyte-Coated Surfaces. J. Colloid Interface Sci. 1998, 205, 77-88. [CrossRef]

5. Shin, Y.; Roberts, J.E.; Santore, M.M. The relationship between polymer/substrate charge density and charge overcompensation by adsorbed polyelectrolyte layers. J. Colloid Interface Sci 2002, 247, 220-230. [CrossRef] [PubMed]

6. Feng, L.; Adachi, Y. Brownian flocculation of negatively charged latex particles with low charge density polycation at various ionic strengths. Colloids Surfaces A Physicochem. Eng. Asp. 2014, 454, 128-134. [CrossRef]

7. Hasan, A.; Fatehi, P. Flocculation of kaolin particles with cationic lignin polymers. Sci. Rep. 2019, 9, 2672. [CrossRef]

8. Lindquist, G.M.; Stratton, R.A. The role of polyelectrolyte charge density and molecular weight on the adsorption and flocculation of colloidal silica with polyethylenimine. J. Colloid Interface Sci. 1976, 55, 45-59. [CrossRef]

9. Aoki, K.; Adachi, Y. Kinetics of polyelectrolyte adsorption onto polystyrene latex particle studied using electrophoresis: Effects of molecular weight and ionic strength. J. Colloid Interface Sci. 2006, 300, 69-77. [CrossRef]

10. Linse, P. Adsorption of Weakly Charged Polyelectrolytes at Oppositely Charged Surfaces. Macromolecules 1996, 29 , 326-336. [CrossRef]

11. Tjipangandjara, K.F.; Somasundaran, P. Effects of changes in adsorbed polyacrylic acid conformation on alumina flocculation. Colloids Surfaces 1991, 55, 245-255. [CrossRef]

12. Ishiduki, K.; Esumi, K. Adsorption Characteristics of Poly(acrylic acid) and Poly(vinyl pyrrolidone) on Alumina from Their Mixtures in Aqueous Solution. J. Colloid Interface Sci. 1997, 185, 274-277. [CrossRef]

13. Yu, X.; Somasundaran, P. Role of Polymer Conformation in Interparticle-Bridging Dominated Flocculation. J. Colloid Interface Sci. 1996, 177, 283-287. [CrossRef]

14. Schwarz, S.; Bratskaya, S.; Jaeger, W.; Paulke, B.R. Effect of charge density, molecular weight, and hydrophobicity on polycations adsorption and flocculation of polystyrene latices and silica. J. Appl. Polym. Sci. 2006, 101, 3422-3429. [CrossRef]

15. Lapointe, M.; Barbeau, B. Understanding the roles and characterizing the intrinsic properties of synthetic vs. natural polymers o improve clarification through interparticle Bridging: A review. Sep. Purif. Technol. 2020, 231, 115893. [CrossRef]

16. Kawaguchi, M. Sequential polymer adsorption: Competition and displacement process. Adv. Colloid Interface Sci 1990, $32,1-41$. [CrossRef]

17. Feng, L.; Stuart, M.C.; Adachi, Y. Dynamics of polyelectrolyte adsorption and colloidal flocculation upon mixing studied using mono-dispersed polystyrene latex particles. Adv. Colloid Interface Sci 2015, 226, 101-114. [CrossRef] [PubMed]

18. Brynda, M.; Chodanowski, P.; Stoll, S. Polyelectrolyte-particle complex formation. Polyelectrolyte linear charge density and ionic concentration effects. Monte Carlo simulations. Colloid Polym. Sci. 2002, 280, 789-797. [CrossRef]

19. De Witt, J.A.; Van de Ven, T.G.M. Kinetics and reversibility of the adsorption of poly(vinyl alcohol) onto polystyrene latex particles. Langmuir 1992, 8, 788-793. [CrossRef]

20. Einarson, M.; Aksberg, R.; Ödberg, L.; Berg, J.C. Adsorption and reconformation of a series of cationic polyacrylamides on charged surfaces. Colloids Surfaces 1991, 53, 183-191. [CrossRef]

21. Kohay, H.; Bilkis, I.I.; Mishael, Y.G. Effect of polycation charge density on polymer conformation at the clay surface and consequently on pharmaceutical binding. J. Colloid Interface Sci. 2019, 552, 517-527. [CrossRef]

22. Rojas, O.J.; Ernstsson, M.; Neuman, R.D.; Claesson, P.M. Effect of Polyelectrolyte Charge Density on the Adsorption and Desorption Behavior on Mica. Langmuir 2002, 18, 1604-1612. [CrossRef]

23. Plunkett, M.A.; Claesson, P.M.; Ernstsson, M.; Rutland, M.W. Comparison of the Adsorption of Different Charge Density Polyelectrolytes: A Quartz Crystal Microbalance and X-ray Photoelectron Spectroscopy Study. Langmuir 2003, 19, $4673-4681$. [CrossRef]

24. Barany, S.; Nagy, M.; Skvarla, J. Electrokinetic potential of polystyrene particles in polyelectrolyte and polyelectrolyte mixtures solutions. Colloids Surfaces A Physicochem. Eng. Asp. 2012, 413, 200-207. [CrossRef]

25. Dukhin, S.S.; Zimmermann, R.; Werner, C. Charge density distribution at interfaces between polyelectrolyte layers and aqueous solutions-Experimental access and limitations of traditional electrokinetics. J. Colloid Interface Sci. 2008, 328, 186. [CrossRef]

26. Adachi, Y.; Kusaka, Y.; Kobayashi, A. Transient behavior of adsorbing/adsorbed polyelectrolytes on the surface of colloidal particles studied by means of trajectory analysis of Brownian motion. Colloids Surfaces A Physicochem. Eng. Asp. 2011, $376,9-13$. [CrossRef]

27. Doan, T.H.Y.; Adachi, Y. Relaxation of adsorbed layer thickness and electrophoresis of polystyrene latex particles after overshooting of polyelectrolytes with different charge density. Colloids Surfaces A Physicochem. Eng. Asp. 2020, 603, 125208. [CrossRef]

28. Kusaka, Y.; Adachi, Y. Determination of hydrodynamic diameter of small flocs by means of direct movie analysis of Brownian motion. Colloids Surfaces A Physicochem. Eng. Asp. 2007, 306, 166-170. [CrossRef]

29. Holde, K.E. Viscosity. In Physical Biochemistry; Prentice-Hall Inc.: Hoboken, NJ, USA, 1971; pp. $166-168$. 
30. Adachi, Y.; Feng, L.; Kobayashi, M. Kinetics of flocculation of polystyrene latex particles in the mixing flow induced with high charge density polycation near the isoelectric point. Colloids Surfaces A Physicochem. Eng. Asp. 2015, 471, 38-44. [CrossRef]

31. Yu, X.; Somasundaran, P. Enhanced flocculation with double flocculants. Colloids Surfaces A Physicochem. Eng. Asp. 1993, 81, 17-23. [CrossRef]

32. Gregory, J.; Barany, S. Adsorption and flocculation by polymers and polymer mixtures. Adv. Colloid Interface Sci 2011, 169, 1-12 [CrossRef]

33. Iruthayaraj, J.; Poptoshev, E.; Vareikis, A.; Makuška, R.; van der Wal, A.; Claesson, P.M. Adsorption of Low Charge Density Polyelectrolyte Containing Poly(ethylene oxide) Side Chains on Silica: Effects of Ionic Strength and pH. Macromolecules 2005, 38, 6152-6160. [CrossRef]

34. Pefferkorn, E.; Elaissari, A. Adsorption-desorption processes in charged polymer/colloid systems; structural relaxation of adsorbed macromolecules. J. Colloid Interface Sci. 1990, 138, 187-194. [CrossRef]

35. Matsumoto, Y.A. and T. Dynamics of initial stage flocculation of polystyrene latex spheres with polyelectrolytes. Colloids Surfaces A Physicochem. Eng. Asp. 1996, 113, 229-236.

36. Van de Steeg, H.G.M.; Cohen Stuart, M.A.; De Keizer, A.; Bijsterbosch, B.H. Polyelectrolyte adsorption: A subtle balance of forces. Langmuir 1992, 8, 2538-2546. [CrossRef]

37. Popa, I.; Gillies, G.; Papastavrou, G.; Borkovec, M. Attractive and Repulsive Electrostatic Forces between Positively Charged Latex Particles in the Presence of Anionic Linear Polyelectrolytes. J. Phys. Chem. B 2010, 114, 3170-3177. [CrossRef]

38. Lyklema, J.; Deschenes, L. The first step in layer-by-layer deposition: Electrostatics and/or non-electrostatics? Adv. Colloid Interface Sci 2011, 168, 135-148. [CrossRef] [PubMed]

39. Gärdlund, L.; Wågberg, L.; Norgren, M. New insights into the structure of polyelectrolyte complexes. J. Colloid Interface Sci. 2007, 312, 237-246. [CrossRef]

40. Shubin, V.; Linse, P. Effect of Electrolytes on Adsorption of Cationic Polyacrylamide on Silica: Ellipsometric Study and Theoretical Modeling. J. Phys. Chem. 1995, 99, 1285-1291. [CrossRef]

41. Bolto, B.; Gregory, J. Organic polyelectrolytes in water treatment. Water Res. 2007, 41, 2301-2324. [CrossRef] [PubMed]

42. Ohshima, H.; Tamotsu, K. Approximate analytic expression for the electrophoretic mobility of colloidal particles with surfacecharge layers. J. Colloid Interface Sci. 1989, 130, 281-282. [CrossRef]

43. Ohshima, H. Approximate Analytic Expression for the Electrophoretic Mobility of Moderately Charged Cylindrical Colloidal Particles. Langmuir 2015, 31, 13633-13638. [CrossRef]

44. Ohshima, H. Approximate Analytic Expression for the Electrophoretic Mobility of a Spherical Colloidal Particle. J. Colloid Interface Sci. 2001, 239, 587-590. [CrossRef] [PubMed]

45. Ohshima, H.; Nakamura, M.; Kondo, T. Electrophoretic mobility of colloidal particles coated with a layer of adsorbed polymers. Colloid Polym. Sci. 1992, 270, 873-877. [CrossRef]

46. Ohshima, H.; Kondo, T. Electrophoresis of large colloidal particles with surface charge layers. Position of the slipping plane and surface layer thickness. Colloid Polym. Sci. 1986, 264, 1080-1084.

47. Ohshima, H.; Kondo, T. Electrophoretic mobility and Donnan potential of a large colloidal particle with a surface charge layer. $J$. Colloid Interface Sci. 1987, 116, 305-311. 